\title{
Espécies de Scolelepis (Polychaeta, Spionidae) de praias do Estado do Rio de Janeiro, Brasil
}

\author{
Marcelo Borges Rocha ${ }^{1,4}$, Vasily Radashevsky ${ }^{2} \&$ Paulo Cesar Paiva ${ }^{3}$ \\ ${ }^{1}$ Programa de Pós-graduação em Zoologia, Museu Nacional, \\ Universidade Federal do Rio de Janeiro - UFRJ, \\ CEP 21941-590, Rio de Janeiro, RJ, Brasil \\ ${ }^{2}$ A.V. Zhirmunsky Institute of Marine Biology \\ Far Eastern Branch of the Russian Academy of Sciences, Vladivostok 690041, Rússia \\ Núcleo de Pesquisa Sócio-Ambientais de Macaé. NUPEM-UFRJ \\ ${ }^{3}$ Departamento de Zoologia, Instituto de Ciências Biológicas - IB, \\ Universidade Federal do Rio de Janeiro - UFRJ, \\ CEP 21941-590, Rio de Janeiro, RJ, Brasil \\ ${ }^{4}$ Autor para correspondência: Marcelo Borges Rocha,e-mail: rochamarcelo36@yahoo.com.br
}

ROCHA, M.B., RADASHEVSKY, V. \& PAIVA, P.C. Species of Scolelepis (Polychaeta, Spionidae) from the beaches of the State of Rio de Janeiro, Brazil. Biota Neotrop., 9(4): http://www.biotaneotropica.org.br/v9n4/ en/abstract?short-communication+bn03309042009.

Abstract: Spionidae is one of the most abundant and diverse taxa among polychaetes. Scolelepis is one of the most abundant genus in shallow waters being widely distributed worldwide. Seven Scolelepis species have been reported to the Brazilian coast, but their taxonomy should be verified owing to a discussion on correct species identification and the possibility of being a species complex. The aim of this study was to make an inventory the species of the genus Scolelepis found in the beaches of Rio de Janeiro State, Brazil. Two species were found: S. chiliensis, very abundant and occurring in several beaches and $S$. goodbodyi with a more restricted distribution.

Keywords: geographic distribution, Southeastern Brazil, soft-bottoms.

ROCHA, M.B., RADASHEVSKY, V. \& PAIVA, P.C. Espécies de Scolelepis (Polychaeta, Spionidae) de praias do Estado do Rio de Janeiro, Brasil. Biota Neotrop., 9(4): http://www.biotaneotropica.org.br/v9n4/en/abstract?shortcommunication+bn03309042009.

Resumo: Entre os poliquetas, os Spionidae são um dos táxons mais abundantes e diversos. O gênero Scolelepis é um dos mais abundantes em águas rasas, cujos representantes apresentam uma ampla distribuição geográfica. Embora tenham sido referidas sete espécies para a costa brasileira, há uma discussão quanto à correta identificação e a possibilidade de se tratar de um complexo específico. O objetivo deste trabalho foi efetuar o levantamento das espécies do gênero Scolelepis encontradas em praias do estado do Rio de Janeiro, Brasil. Foram encontradas e caracterizadas duas espécies: S. chilensis, muito abundante e ocorrendo em diversas praias e S. goodbodyi com uma distribuição mais restrita.

Palavras-chave: distribuição geográfica, sudeste brasileiro, substrato inconsolidado. 


\section{Introdução}

Spionidae Grube, 1850 é uma das famílias mais ricas e abundantes dentro de Polychaeta com cerca de 500 espécies distribuídas em 33 gêneros atualmente reconhecidos. $\mathrm{O}$ grupo tem uma vasta distribuição mundial, ocorrendo em uma ampla faixa batimétrica e em quase todos os tipos de substratos (Blake 1996). Dentre os gêneros de Spionidae, Scolelepis é um dos que apresenta maior número de espécies ocorrendo principalmente em ambientes de praias. Sua ampla distribuição tem sido atribuída às características morfológicas que lhes permitem adaptar-se bem a este tipo de ambiente. Dentre estas características, se destacam a presença de um prostômio acuminado, que facilita o escavamento rápido no substrato arenoso e de palpos longos capazes de capturar alimento em diferentes condições, tanto depositados quanto em suspensão, permitindo assim uma grande capacidade de colonizar áreas praiais (Pardo \& Amaral 2004).

O gênero Scolelepis foi revisto por Maciolek (1987) que propôs o estabelecimento de dois subgêneros, Scolelepis e Parascolelepis com base na morfologia dos dentes dos ganchos. O subgênero Scolelepis De Blainville 1828 incluiria as espécies com ganchos uni-, bi- ou tridentados, enquanto Parascolelepis Maciolek 1987 incluiria espécies com ganchos multidentados. Nesse estudo, será adotada a classificação apenas em nível genérico visto que não há estudos suficientes que comprovem o monofiletismo dos supostos sub-gêneros.

Atualmente são reconhecidas, em todo o mundo, quase 80 espécies de Scolelepis. Para a costa brasileira foram referidas nominalmente, até o momento, sete espécies: Scolelepis chilensis HartmannSchröder, 1962; S. goodbodyi Jones, 1962; S. squamata Muller, 1806; S. texana Foster, 1971 (Amaral et al. 2006), S. lighti Delgado-Blas, 2006 (Santos et al. 2008), Scolelepis quinquedentata HartmannSchröder, 1965 (Lana et al. 2006) e S. gaucha Orensanz-Gianuca, 1974 descrita originalmente como Spio gaucha. Para o litoral do Rio de Janeiro há registros publicados de S. squamata (Fernandes \& Soares-Gomes 2006) e S. texana (Amaral \& Rossi-Wongtschowski 2004). Não obstante as espécies supracitadas, não há ainda uma avaliação clara sobre a real ocorrência de todas elas, principalmente quando se considera a grande variabilidade morfológica encontrada em algumas delas. Dentre as espécies, a que apresenta o maior número de registros e uma ampla distribuição ao longo da costa brasileira é Scolelepis squamata. No entanto, esta é uma das espécies de poliquetas tidas como cosmopolitas e que certamente requer uma revisão taxonômica. Estudos recentes constataram que existe uma grande variabilidade de morfotipos no material originalmente referido a S. squamata ao longo da costa brasileira (Bolívar \& Lana 1986). Por exemplo, espécimes antes referidos a $S$. squamata, no litoral norte do estado de São Paulo foram recentemente identificadas como duas espécies distintas (MacCord \& Amaral 2005): Scolelepis goodbodyi Jones, 1962 e Scolelepis cf. chilensis Hartmann-Schröder, 1962.

O objetivo deste trabalho foi efetuar um levantamento e caracterização das espécies do gênero Scolelepis em praias do estado do Rio de Janeiro, Brasil.

\section{Material e Métodos}

Os espécimes foram coletados nas seguintes localidades: 1) Praia de Dois Rios, localizada na Ilha Grande, Angra dos Reis, no sul do

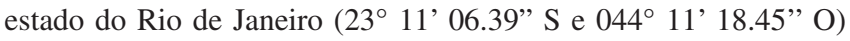
em julho de 2000; 2) Praia do Flamengo, localizada no município do Rio de Janeiro, na costa central do estado $\left(22^{\circ} 55^{\prime} 24.40^{\prime \prime} \mathrm{S}\right.$ e $043^{\circ} 10^{\prime} 06.23^{\prime}$ ' O), em junho de 2005, abril de 2007 e março de 2008; 3) Ilha de Marambaia localizada na Baía de Sepetiba (22 $51^{\prime}$ '07.36" S e 043 $57^{\circ} 11.59$ " O) em janeiro de 2004, também na costa central do Estado e 4) Praia de Manguinhos, localizada no muni- cípio de Armação dos Búzios (22 45’ 48.21"' S e 041 ${ }^{\circ} 55^{\prime} 01.01$ ” O) em julho de 2009.

O material foi fixado em solução de formaldeído $10 \%$ e posteriormente transferido para etanol $70 \%$ para a preservação de longo prazo. Os espécimes foram examinados sob microscópio estereoscópico no Laboratório de Polychaeta da Universidade Federal do Rio de Janeiro. Para uma análise completa do material, espécimes completos e partes foram preparados em lâminas permanentes com gelatina/glicerina (Amaral \& Nonato 1994). As ilustrações da morfologia externa foram confeccionadas sob câmara clara acoplada a microscópio estereoscópico e microscópio óptico.

Foram também efetuadas observações, de dois indivíduos da Praia de Dois Rios e de três indivíduos da Praia de Manguinhos em Microscópio Eletrônico de Varredura (Jeol JSM 6390 LV) com o ponto crítico sendo realizado em um CPD 030 (Leica) e metalização a ouro no equipamento Balzers. Devido à presença de capuz nos ganchos, para a observação destes em MEV foi utilizado um sonicador de $40 \mathrm{~Hz}$, que através de ondas de ultra-som remove o capuz. Para as observações em MEV, as amostras foram transferidas do álcool 70\% para a água destilada por uma hora, a fim de substituir o etanol do tecido corporal. $\mathrm{O}$ material foi cortado em fragmentos e estes foram sonicados em água destilada por 40 segundos. Depois desse banho, os fragmentos foram desidratados com uma série de etanol de 50, 70, 80,90 e $100 \%$, ficando 10 minutos. em cada concentração. O material foi então observado em Microscópio Eletrônico de Varredura no Instituto de Biofísica da Universidade Federal do Rio de Janeiro e no Museu Nacional - UFRJ.

Todo o material foi tombado na Coleção de Poliquetas 'Edmundo Ferraz Nonato' do Instituto de Biologia da Universidade Federal do Rio de Janeiro (IBUFRJ).

\section{Resultados}

Análises morfológicas permitiram a identificação de duas espécies do gênero: $S$. goodbodyi e $S$. chilensis.

\section{Scolelepis goodbodyi (Jones, 1962) (Figuras 1 e 2)}

Nerinides goodbodyi Jones, 1962: 187, figs. 66-68.

Scolelepis goodbodyi ' - Delgado-Blas, 2006:83, fig. 4.

Material examinado: 22 espécimes: IBUFRJ - 0730 (10), $22^{\circ} 55^{\prime} 24.40^{\prime}$ S e $043^{\circ} 10^{\prime} 06.23$ " O, Praia do Flamengo, Rio de Janeiro, 15 vi 2005; IBUFRJ-0736 (10), 23 11'06.39”' S e $44^{\circ} 11$ ' 18.45" O, Praia de Dois Rios, Ilha Grande, Angra dos Reis, Rio de Janeiro, 10 vii 2000; IBUFRJ - 0737 (2, MEV), $23^{\circ} 11$ '06.39" S e 44 11' 18.45" O, Praia de Dois Rios, Ilha Grande, Angra dos Reis, Rio de Janeiro, 10 vii 2005.

Caracterização: Foram observados 19 espécimes completos 10-15 mm de comprimento, 0,5-0,6 mm de largura no setígero 2, com 56-71 setígeros e três espécimes incompletos 11-12 mm de comprimento, 0,6-0,7 mm de largura, com 45-48 setígeros. Coloração amarela em álcool. Prostômio acuminado e alongado, duas vezes mais longo do que largo, com as extremidades afiladas (Figuras 2a-d), alargando-se na altura do peristômio, assumindo um formato triangular (Figura 1a). Carúncula presente se estendendo até o $1^{\circ}$ setígero (Figura 2 b). Peristômio longo e com asas laterais pouco desenvolvidas (Figura 2c). Dois pares de olhos marrom-avermelhados dispostos em linha transversal. Olhos centrais arredondados e olhos laterais reniformes. Sem qualquer indício de pigmentação secundária na região dos olhos. Palpos longos atingindo o setígero 21.

Brânquias a partir do $2^{\circ}$ setígero, afiladas e alongadas, sobretudo nas regiões anterior e mediana do corpo. Brânquias parcialmente fundidas, cerca de $1 / 2$, às lamelas notopodiais até 

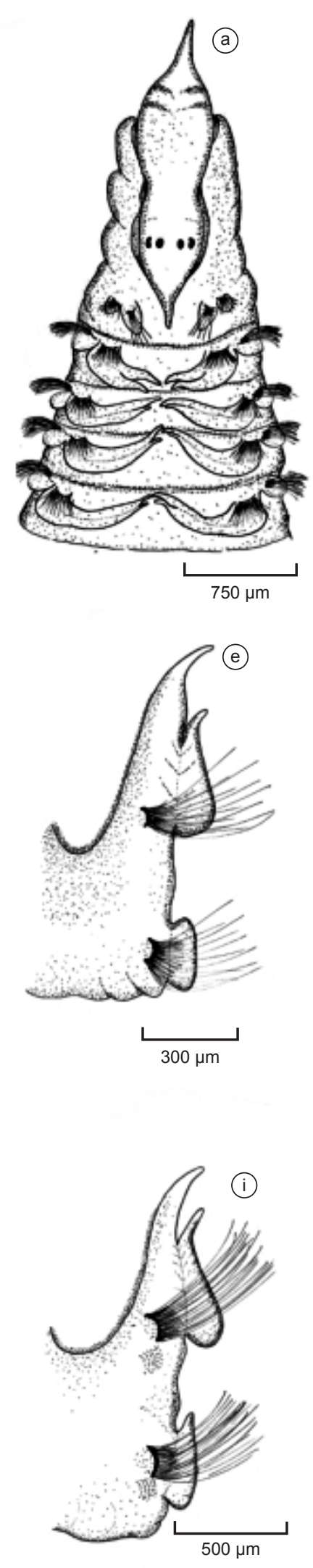

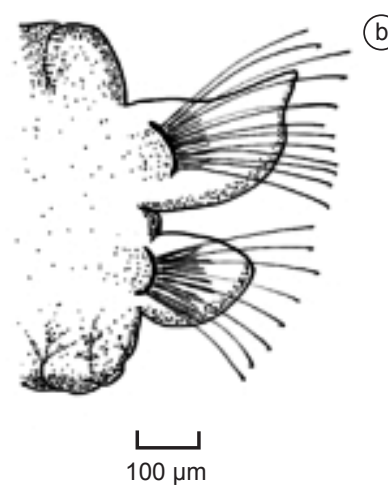

(b)
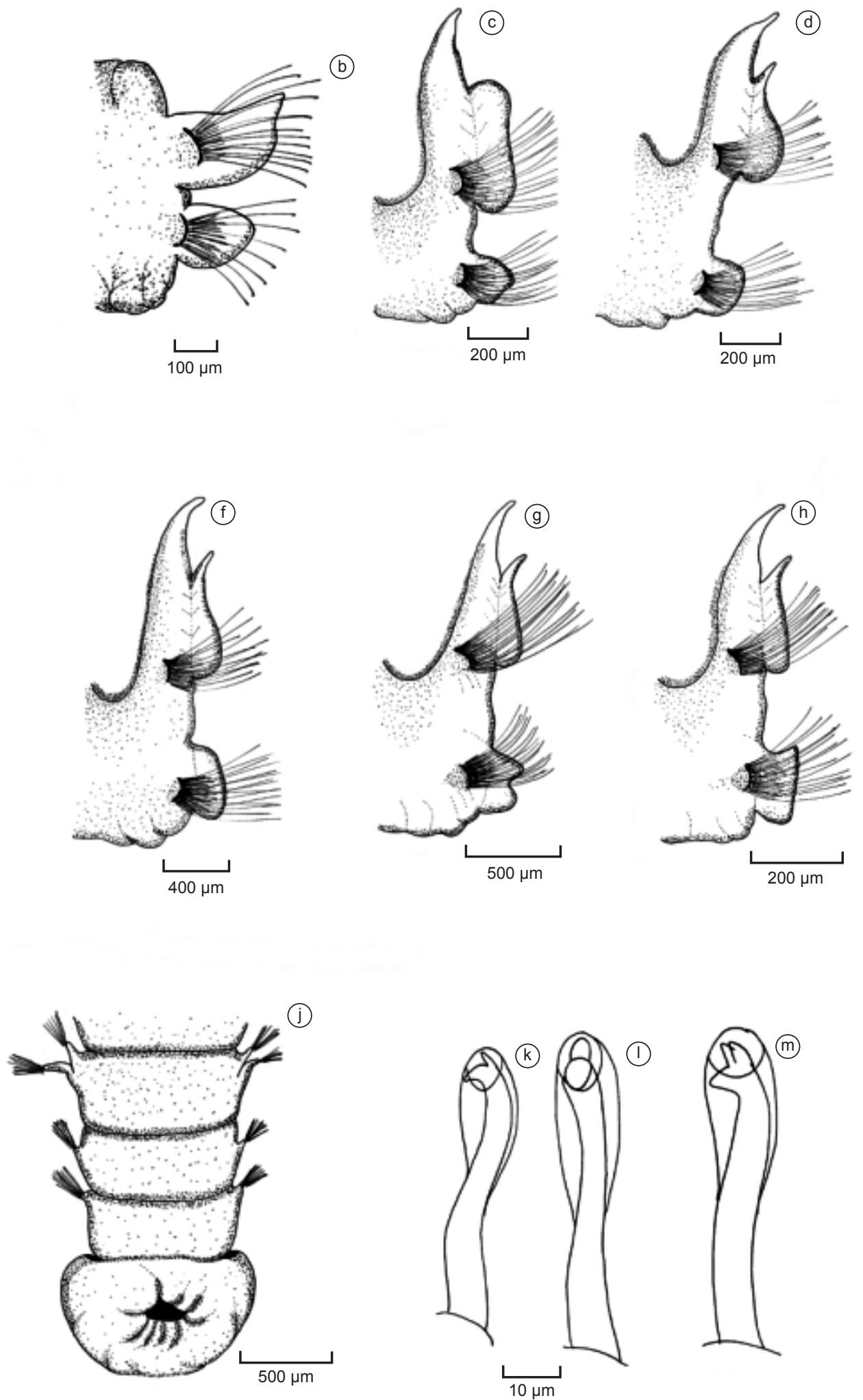

Figura 1. Scolelepis goodbodyi. a) Região anterior, vista dorsal. b) Parapódio do setígero 1. c) Parapódio do setígero 2. d) Parapódio do setígero 10. e) Parapódio do setígero 16. f) Parapódio do setígero 20. g) Parapódio do setígero 22. h) Parapódio do setígero 30. i) Parapódio do setígero 40. j) Pigídio. k-m) Ganchos neuropodiais dos setígeros 35,40 e 65 .

Figure 1. Scolelepis goodbodyi. a) Anterior region, dorsal view. b) Parapodium of chaetiger 1 c) Parapodium of chaetiger 2. d) Parapodium of chaetiger 10. e) Parapodium of chaetiger 16. f) Parapodium of chaetiger 20. g) Parapodium of chaetiger 22. h) Parapodium of chaetiger o 30. i) Parapodium of chaetiger 40. j) Pygidium. k-m) Neuropodial hooks of chaetigers 35, 40 e 65. 

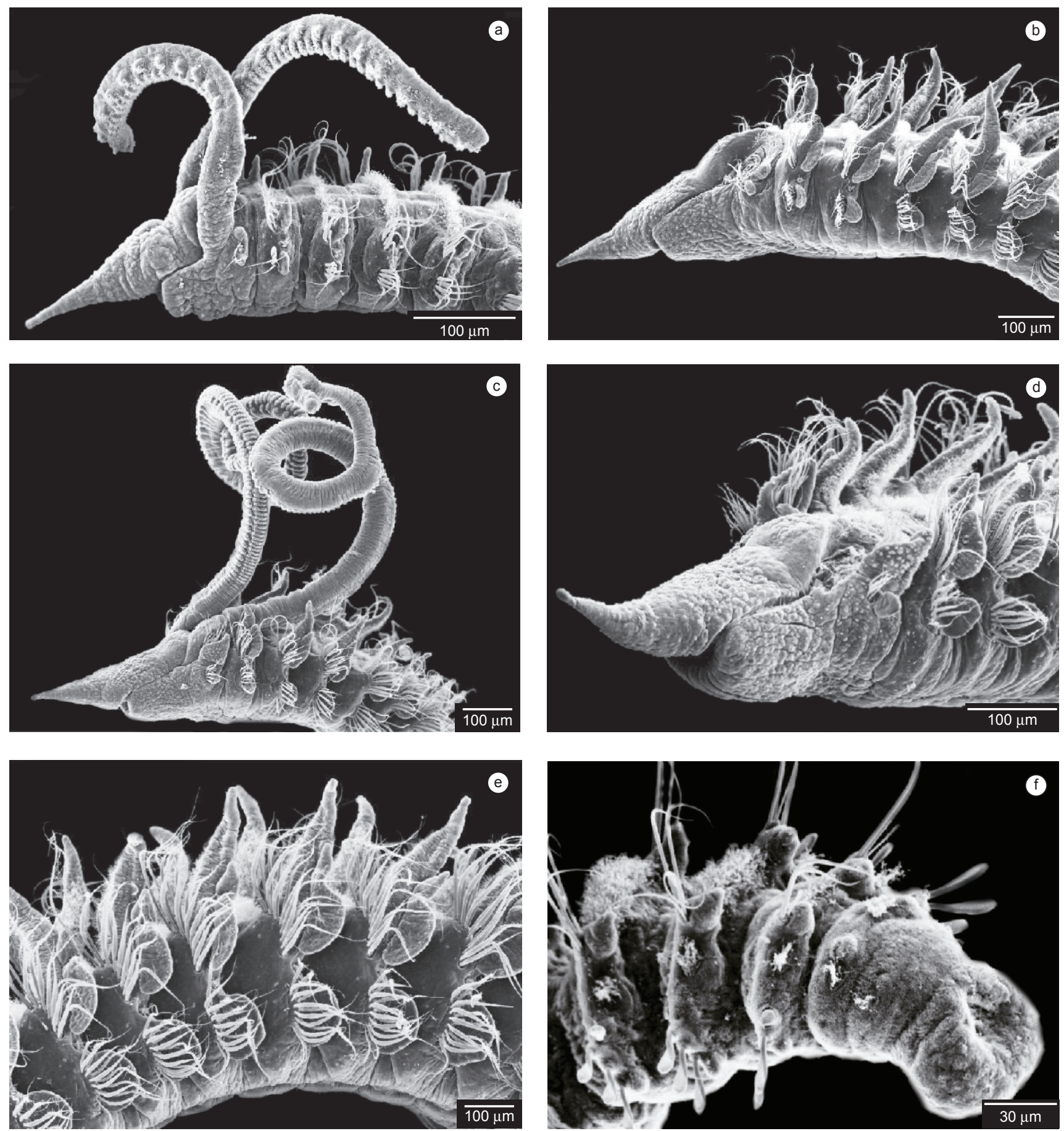

Figura 2. Scolelepis goodbodyi. a) Região anterior de um indivíduo pequeno (10 mm), com palpos curtos, vista lateral esquerda, mostrando setígero 1 com lamelas notopodiais pequenas e notocerdas ainda não desenvolvidas. b) Extremidade anterior de um indivíduo mediano (12 mm), sem palpos, vista lateral esquerda, mostrando setígero 1 com lamelas notopodiais pequenas. c) Extremidade anterior de um indivíduo grande (15 mm), com palpos longos, vista lateral esquerda, mostrando setígero 1 com lamelas bem desenvolvidas e capilares notopodiais dorsais. d) Extremidade anterior de um indivíduo grande, sem palpos, vista lateral esquerda, mostrando setígero 1 com lamelas bem desenvolvidas e capilares notopodiais dorsais. e) Setígeros 6-11, vista lateral esquerda, mostrando lamelas notopodiais fundidas às brânquias, e todas as lamelas neuropodiais, semi-ovais. f) Extremidade posterior vista lateral esquerda, mostrando pigídio com formato de almofada.

Figure 2. Scolelepis goodbodyi. a) Anterior end of a small individual $(10 \mathrm{~mm})$, short palps, left lateral view, showing chaetiger 1 with small notopodial lamellae and notochaetae not yet developed. b) Anterior end of a median individual $(12 \mathrm{~mm})$, palps missing, left lateral view, showing chaetiger 1 with small notopodial lamellae. c) Anterior end of a large individual $(15 \mathrm{~mm})$ with long palps, left lateral view, showing chaetiger 1 with well developed notopodial lamellae and dorsal capillaries. d) Anterior end of a large individual, palps missing, left lateral view, showing chaetiger 1 with well developed notopodial lamellae and dorsal capillaries. e) Chaetigers 6-11, left lateral view, showing notopodial postchaetal lamellae fused to branchiae, and neuropodial postchaetal lamellae entire, semi-oval. f) Posterior end, left lateral view, showing small cushion-like pygidium. 
aproximadamente o setígero 22, a partir daí, tornam-se fundidas apenas, cerca de 1/3, na região basal da lamela (Figuras 1d, 1e, 2e). Brânquias com bandas ciliares ao longo de toda a extensão. Células glandulares presentes na região mediana das brânquias entre os setígeros 16 e 22 (Figura 1f).

Primeiro parapódio bem desenvolvido com lamelas de diferentes formatos: triangular no notopódio e arredondada no neuropódio (Figura 1b). Segundo setígero apresenta lamela notopodial quadrada com um leve truncamento na ponta (Figura. 1c). Os demais setígeros apresentam lamelas notopodiais alongadas e afiladas (Figura 1d-i).

Lamelas neuropodiais arredondadas nos setígeros iniciais, tornando-se retangulares ou levemente quadradas a partir do setígero 16 (Figuras 1e, 1f) e triangular a partir aproximadamente do setígero 26 (Figuras 1h, 1j). Entre os setígeros 16 e 19 há uma transição no formato das lamelas neuropodiais, que se tornam bilobadas com lobos subiguais (Figura 1g). Neuropódios dos setígeros anteriores e medianos com cerdas capilares apenas. Ganchos neuropodiais aparecem entre os setígeros 24 e 27 em número de sete a nove por neuropódio. Os ganchos apresentam um ou dois dentes menores posicionados lado a lado sobre o dente principal e um capuz externo (Figuras 1k, 1m).

Pigídio arredondado em forma de almofada. (Figuras $1 \mathrm{j}$ e 2f)

Distribuição: A espécie foi descrita originalmente a partir de material coletado na Jamaica (Mar do Caribe), sendo posteriormente registrada para os estados de São Paulo e Paraná na costa sul do Brasil (MacCord \& Amaral 2005, Lana et al. 2006). Este é o primeiro registro para o estado do Rio de Janeiro.

2. Scolelepis chilensis Hartmann-Schröder, 1962 (Figuras 3 e 4)

Nerine cirratulus chilensis Hartmann-Schröder, 1962: 142-144, figs. 179-182

Scolelepis chilensis - Blake, 1983:207, fig. 2.

Material examinado: 32 espécimes: IBUFRJ - 0731 (6), $22^{\circ} 55^{\prime} 24.40^{\prime \prime} \mathrm{S}$ e $043^{\circ} 10^{\prime} 06.23^{\prime \prime} \mathrm{O}$, Praia do Flamengo, Rio de Janeiro, 07 vi 2005; IBUFRJ - 0732 (3), 22 $55^{\prime}$ '24.40' $\mathrm{S}$ e $043^{\circ} 10^{\prime}$ 06.23"' O, Praia do Flamengo, Rio de Janeiro, 04 iv 2007; IBUFRJ - 0733 (4), $22^{\circ}$ 55' 24.40”'S e 043 10'06.23" O, Praia do Flamengo, Rio de Janeiro, 03 iii 2008; IBUFRJ - 0734 (5), $22^{\circ} 45$ ' 48.21" S e $041^{\circ} 55^{\prime}$ 01.01" O, Praia de Manguinhos, Armação dos Búzios, Rio de Janeiro, 02 vi 2009; IBUFRJ - 0735 (14), 22 51'07.36" S e 043 57' 11.59”' O, Baía de Sepetiba Ilha de Marambaia, Rio de Janeiro, 12 i 2004.

Caracterização: Foram examinados 26 espécimes completos, 7-18 mm de comprimento, com 0,6-1,2 mm de largura no setígero 2, com 63-97 setígeros e seis espécimes incompletos, de 6-12 mm de comprimento, 0,7-0,9 mm de largura, com 32-46 setígeros. Cor marrom claro em álcool. Prostômio alongado e acuminado, duas vezes mais longo do que largo. Carúncula bem desenvolvida estendendo-se até o setígero 1 (Figura 4a). Peristômio bem desenvolvido com asas laterais que não chegam a envolver o prostômio. Dois pares de olhos marrons arranjados em linha transversal. Olhos centrais arredondados e olhos laterais reniformes quando observados dorsalmente devido a sua posição lateral contrastar com a ventral (Figura 3a). Possui duas bandas de pigmentação avermelhada sobre os olhos. Palpos atingindo o $20^{\circ}$ setígero providos de duas bandas ciliares (Figura $4 \mathrm{~b}$ ).

Brânquias a partir do $2^{\circ}$ setígero, afiladas e alongadas, sobretudo nas regiões anterior e mediana do corpo e apresentando cílios na face interna. Brânquias parcialmente fundidas, cerca de $1 / 2$, às lamelas notopodiais até o setígero 22, a partir daí, tornam-se fundidas apenas, cerca de $1 / 3$, na região basal da lamela (Figuras 4 d-e).
Primeiro parapódio pouco desenvolvido com lamelas arredondadas e alongadas de diferentes formatos respectivamente no notopódio e no neuropódio (Figura 3b, 4c). Lamelas notopodiais alongadas e ovais a partir do setígero 2 (Figura 3c) com leve reentrância no setígero 7 (Figura 3d). Lamelas neuropodiais arredondadas nos setígeros anteriores tornando-se mais alongadas a partir do setígero 21 (Figuras 3e, 3f, 3g). Presença de duas fileiras ciliadas nos segmentos, sendo uma mais delgada e outra espessa (Figura 4e).

Ganchos neuropodiais aparecem a partir do setígero 25, em número de sete a nove por neuropódio, se estendendo até o último setígero. Ganchos com um, dois ou três dentes menores posicionados lado a lado sobre o dente principal e com um capuz externo (Figuras 3h, 3i).

Pigídio arredondado em forma de almofada (Figuras 3j, 4f).

Distribuição: A espécie foi descrita originalmente a partir de material proveniente do Chile, em região entremarés sendo depois referida para o estado de São Paulo na costa sul do Brasil (MacCord \& Amaral 2005). É possível que ocorra na costa do Paraná, onde foi referida como $S$. squamata, cuja variabilidade intra-populacional levou Bolívar \& Lana (1986) a sugerir se tratar de um complexo específico.

\section{Discussão}

As espécies caracterizadas nesse estudo se assemelham ao material encontrado em outras regiões da costa sul do Brasil que, em passado recente, foram referidas de forma abrangente a $S$. squamata, considerada então como uma espécie de grande variabilidade morfológica (Bolívar \& Lana 1986). Não obstante esta similaridade morfológica entre ambas as espécies, $S$. goodbodyi e $S$. chilensis, e $S$. squamata, estas podem ser diferenciadas morfologicamente a partir de uma análise detalhada.

O material aqui referido a $S$. goodbodyi se aproxima da descrição original de Jones (1962) e da redescrição feita por Delgado-Blas (2006), principalmente pelos caracteres diagnósticos como a presença das lamelas notopodiais bem alongadas e ovais com uma suave ponta em sua extremidade, pela presença de uma pequena reentrância na lamela neuropodial (no setígero 20 na descrição original e entre os setígeros 16-19 em nosso material), pelo prostômio de formato triangular apresentando uma extremidade anterior bem aguda e se estendendo até o $1^{\circ}$ setígero. Além dessas características pode-se destacar ainda como características diagnósticas a presença de células glandulares nas brânquias e o $1^{\circ}$ setígero com a lamela neuropodial arredondada e a notopodial triangular bem desenvolvida. $\mathrm{O}$ formato dos ganchos é similar em ambas as descrições (Jones 1962, Delgado-Blas, 2006) e o número de ganchos por neuropódio é o mesmo (sete-nove). A região onde há o aparecimento dos ganchos também é bastante semelhante, ou seja, entre os setígeros 24 e 27.

Scolelepis goodbodyi é muito similar a S. squamata, tendo sido considerada sinônimo júnior desta espécie pela semelhança da primeira lamela neuropodial e a forma e posição dos olhos. No entanto difere pela ausência de pigmentação nos primeiros setígeros em $S$. goodbodyi, pela extensão do peristômio, que se estende até o $2^{\circ}$ setígero em $S$. squamata e até o $1^{\circ} \mathrm{em} S$. goodbodyi. Além disso, o parapódio do $1^{\circ}$ setígero em $S$. goodbodyi possui a lamela notopodial triangular e a neuropodial arredondada, já S. squamata possui a lamela notopodial oval e a neuropodial arredondada. Diferem ainda quanto à forma das lamelas notopodiais e neuropodiais.

O material referido a $S$. chilensis se aproxima da descrição original de Hartmann-Schröder (1962) e da redescrição feita por Blake (1983) principalmente por apresentar o $1^{\circ}$ parapódio bem reduzido, pela presença de uma reentrância nas lamelas notopodiais a partir 

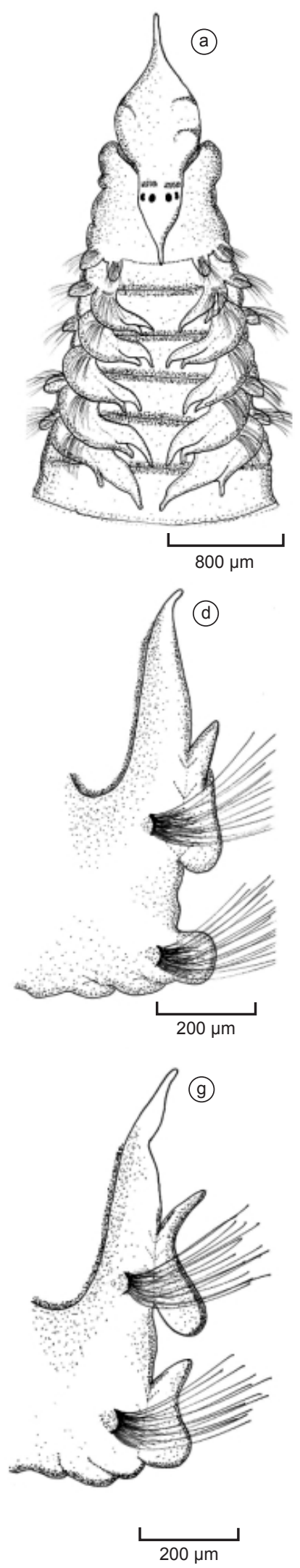
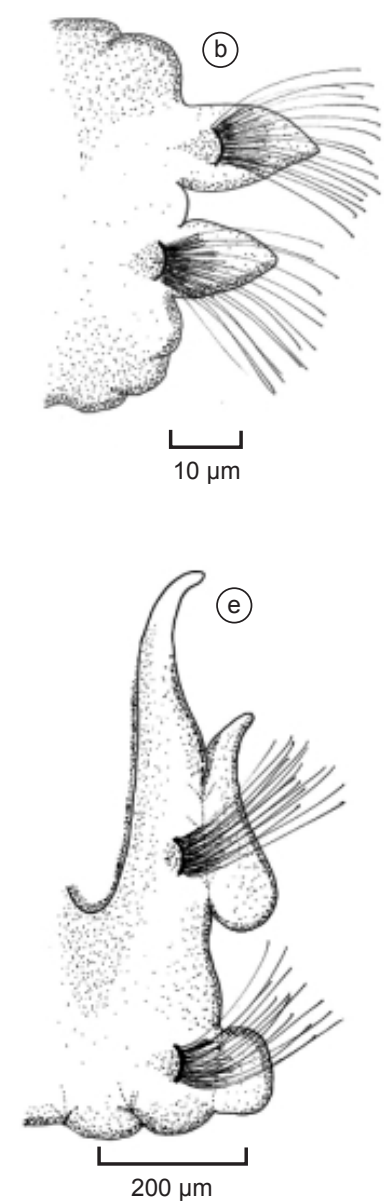
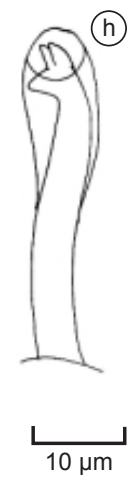
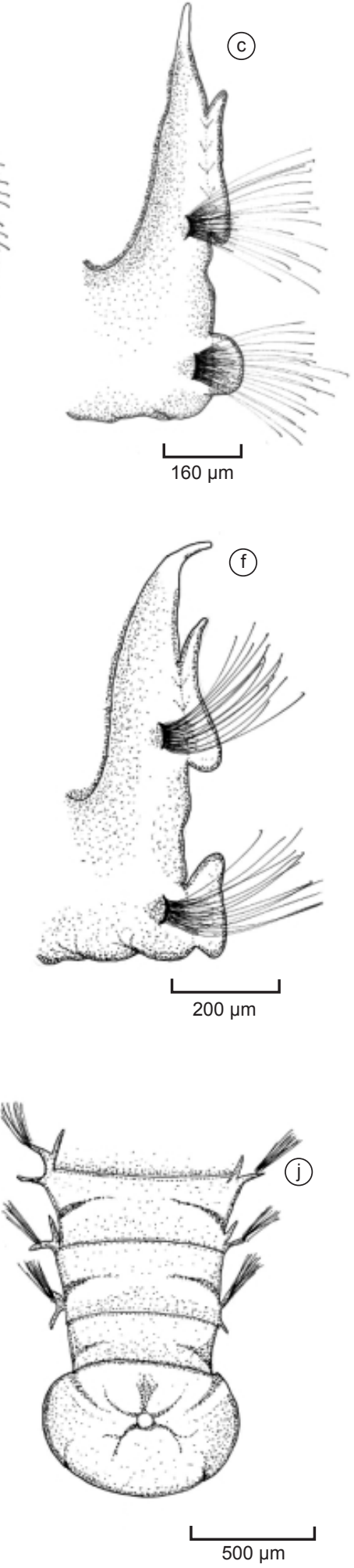

Figura 3. Scolelepis chilensis. a) Região anterior vista dorsal. b) Parapódio do setígero 1. c) Parapódio do setígero 2. d) Parapódio do setígero 7. e) Parapódio do setígero 16. f) Parapódio do setígero 32. g) Parapódio do setígero 40 h-i) Ganchos neuropodiais dos setígeros 36 e 40. j) Pigídio.

Figure 3. Scolelepis chilensis. a) Anterior region, dorsal view 1. b) Parapodium of chaetiger 1. c) Parapodium of chaetiger 2. d) Parapodium of chaetiger 7. e) Parapodium of chaetiger 16. f) Parapodium of chaetiger 32. g) Parapodium of chaetiger 40 h-i) Neuropodial hooks of chaetigers 36 and 40 . j) Pygidium. 

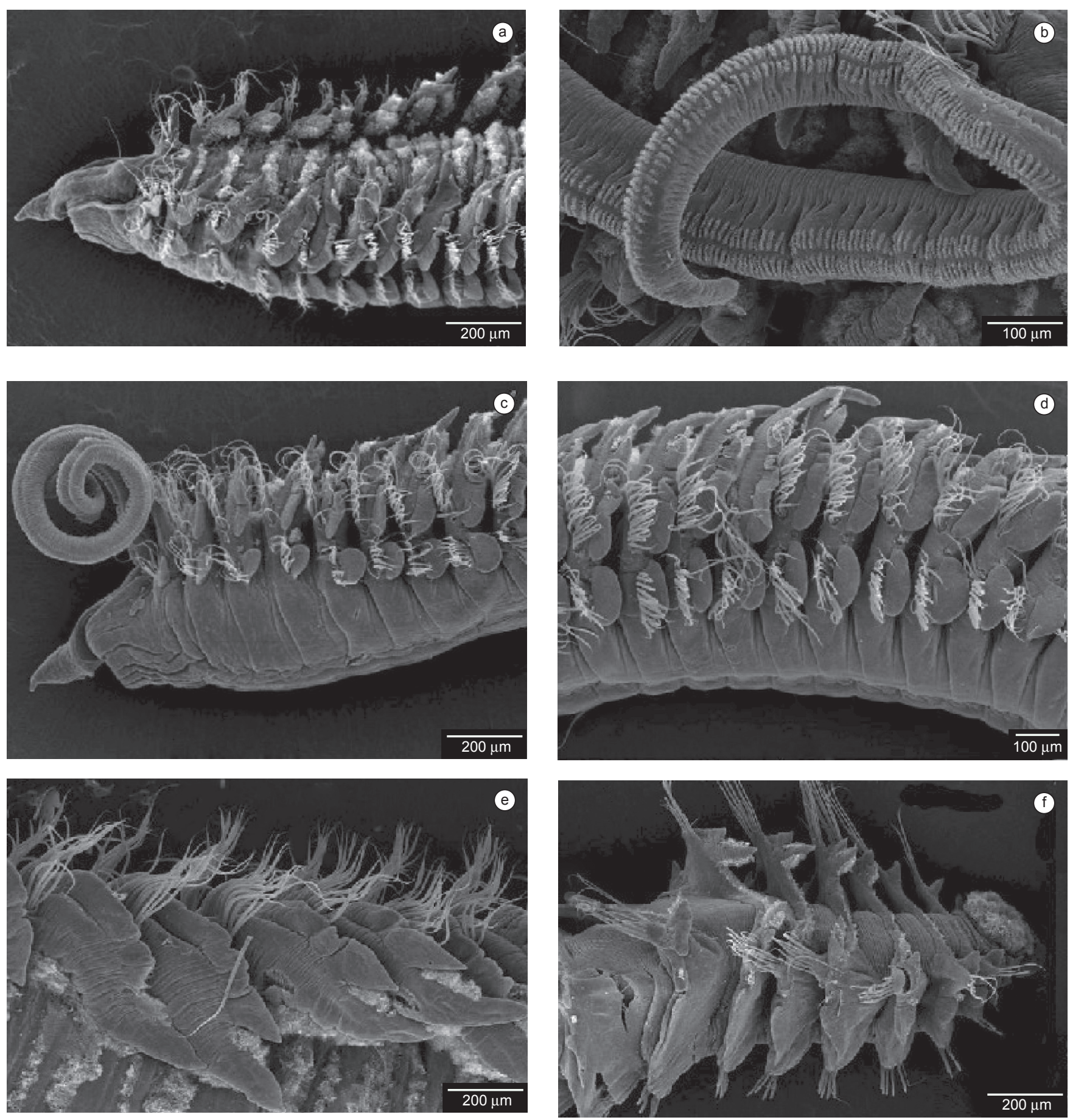

Figura 4. Scolelepis chilensis. a) Região anterior vista latero-dorsal, sem palpos. b) Palpos. c) Região anterior vista lateral com palpo. d) Setígeros 17-26, mostrando o grau de fusão das lamelas notopodiais com as brânquias e lamelas neuropodiais arredondadas. e) Detalhe das brânquias dos setígeros 10-15 mostrando o grau de fusão das brânquias com as lamelas notopodiais. f) Região posterior com vista latero-dorsal mostrando o pigídio em forma de almofada.

Figura 4. Scolelepis chilensis. a) Anterior end, left latero-dorsal view, palps missing. b) Palps. c) Anterior end, left lateral view with palp. d) Chaetigers 17-26, showing notopodial postchaetal lamellae fused to branchiae, and neuropodial postchaetal lamellae entire, rounded. e) Chaetigers 10-15 showing notopodial postchaetal lamellae fused to branchiae. f) Posterior end, showing small cushion-like pygidium.

do $7^{\circ}$ setígero, brânquias pouco alongadas, carúncula bem desenvolvida e peristômio inflado formando asas laterais. Houve uma variação na quantidade de dentes dos ganchos quando comparado ao material examinado por Blake que descreve um dente menor e um principal, enquanto nos espécimes examinados neste trabalho registramos um ou dois menores e um grande. Blake (1983) ao estudar essa espécie descreveu a presença de ganchos bidentados, com um dente principal e outro formado pela conexão através de uma ponte entre dois dentes menores, considerando-o como um único dente. Em nosso estudo foi possível observar, com auxílio da microscopia eletrônica de varredura, a presença de ganchos com dois e três dentes bem distintos. 
Scolelepis chilensis também é próxima de S. squamata pela presença de ganchos bidentados nos segmentos posteriores do corpo e pelo formato da lamela notopodial do $1^{\circ}$ setígero. Porém, em S. chilensis o grau de fusão da lamela notopodial com as brânquias é maior nos primeiros setígeros e o capuz dos ganchos de $S$. chilensis apresenta finas estrias, ausentes no capuz de $S$. squamata. Outra diferença entre essas espécies é o fato de $S$. squamata apresentar ganchos uni-, bi- ou tridentados e $S$. chilensis apenas uni ou bidentados.

Alguns dos espécimes referidos por Bolívar \& Lana (1986) para a costa do Paraná, podem se tratar de S. chilensis, em função da forma das brânquias e das lamelas neuropodiais. Foi possível observar uma considerável variabilidade fenotípica de Scolelepis chilensis nas praias do Rio de Janeiro. Dentre os caracteres que apresentam variação destacam-se o formato do prostômio que variou de alongado no material coletado na Praia do Flamengo a ligeiramente triangular na altura do peristômio nos espécimes da Ilha de Marambaia, as lamelas notopodiais do $1^{\circ}$ setígero apresentaram-se alongadas na Ilha de Marambaia e ligeiramente ovais na Praia do Flamengo.

No presente estudo pode-se observar que uma grande variabilidade morfológica pode mascarar a ocorrência de espécies do gênero Scolelepis dentro de um possível complexo específico.

\section{Agradecimentos}

A todos aqueles que contribuíram nas coletas do material: Bruna Tovar Faro, Danielle Vilela, Ricardo Bastos Guimarães, Christine Ruta e Gabriela Neves de Souza. Esta publicação foi parcialmente financiada com recursos da CAPES pela concessão de bolsa de Professor Visitante Estrangeiro para VIR e do CNPq pela concessão de bolsa de Produtividade em Pesquisa a PCP.

\section{Referências Bibliográficas}

AMARAL, A.C.Z. \& ROSSI-WONGTSCHOWSKI, C.L.D.B. 2004. Biodiversidade bentônica da região sudeste-sul do Brasil: plataforma externa e talude superior. Ulhôa Cintra Ed., São Paulo, p. 1-216. (Série Documentos Revizee - Score Sul)

AMARAL, A.C.Z., NALLIN, S.A.H. \& STEINER, T.M. 2006. Catálogo das espécies dos Annelida Polychaeta do Brasil. São Paulo: Campinas. http://www.ib.unicamp.br/destaques/biota/bentos_marinho/prod_cien/ texto_poli.pdf (último acesso em 25/09/2009).

AMARAL, A.C.Z. \& NONATO, E.F. 1994. Anelídeos poliquetas da costa brasileira. 5. Pisionidae, Chrysopetalidae, Amphinomidae e Euphrosionidae. Rev. Bras. Zool. 11 (2):361-390.
BLAKE, J.A. 1996. Family Spionidae Grube, 1850 In Taxonomic atlas of the Santa Maria Basin and western Santa Barbara Channel: the Annelida (J.A. Blake, B. Hilbig \& P.H. Scott, eds.). Santa Barbara Museum of Natural History, Santa Barbara, p. 81-223. (v. 6, part 3, Polychaeta: Orbiniidae to Cossuridae)

BLAKE, J.A. 1983. Polychaetes of the family Spionidae from South America, Antarctica, and adjacent seas and islands. Antarct. Res. Ser. 39:205-288. (Biology of the Antarctic Seas XV)

BOLIVAR, G.T.A. \& LANA, P.C. 1986. Spionidae (Annelida: Polychaeta) do litoral do Estado do Paraná. Neritica. 1(3):107-148.

DELGADO-BLAS, V. 2006. Partial revision of Scolelepis (Polychaeta:Spionidae) from the Grand Caribbean Region, with the description of two new species and a key to species recorded in the area. Contrib. Zool. 75(2):75-97.

FERNANDES, R.S.R. \& SOARES-GOMES, A. 2006. Community structure of macrobenthos in two tropical sandy beaches with different morphodynamic features, Rio de Janeiro, Brazil. Mar. Biol. 27(2):160-169.

HARTMANN-SCHÖDER, G. 1962. Die polychaeten des Eulitorals. In Zur Kenntnis des Eulitorals der Chilenischen Pazifikküste und der Argentinischen Küste, südpatgoniens unter besonderer Berücksichtigung der Polychaeten und Ostracoden (G. Hartmann-Schöder \& G. Hartmann, eds.). Mitt. Hamb. Zool. Mus. Inst. 60:57-167.

JONES, M.L. 1962. On some polychaetous annelids from Jamaica: the west indies. B. Am. Mus. Nat. Hist. 124(5):173-212.

LANA, P.C., SANTOS, C.S.G., GARRAFFONI, A.R.S., OLIVEIRA, V.M., RADASHEVSKY, V. 2006. Checklist of polychaete species from Paraná State (Southern Brazil). Check List. 2(3):30-63.

MACIOLEK, N.J. 1987. New species and records of Scolelepis (Polychaeta: Spionidae) from the east coast of North America, with a review of the subgenera. Bull. Am. Mus. Nat. Hist. 7:16-40.

MACCORD, F.S. \& AMARAL, A.C.Z. 2005. Morphometric analysis of two species of Scolelepis (Polychaeta: Spionidae). J. Mar. Biol. 84:769-784.

ORENSANZ, J.M. \& GIANUCA, N.M. 1974. Contribuição ao conhecimento dos anelídeos poliquetas do Rio Grande do Sul: lista sistemática preliminar e descrição de três novas espécies. Com. Mus. Ciênc. 4:1-37.

PARDO, E.V. \& AMARAL, A.C.Z. 2004. Feeding behavior of Scolelepis sp. (Polychaeta: Spionidae). Braz. J. Oceanogr. 52(1):75-79.

SANTOS, A.S., COSTA, D.A. \& CHRISTOFFERSEN, M.L. 2008. First record of Scolelepis (Scolelepis) lighti along the Brazilian coast. J. Mar. Biol. Assoc. 2:1-5. 